\title{
The age-specific differences in histopathological tumor characteristics and TNM classification of breast carcinomas in Quality assured mamma diagnostic (QuaMaDi) program in the state of Schleswig-Holstein in Germany
}

\author{
L.-J. Kramp ${ }^{1}$ M. Mathiak ${ }^{1}$ H.-M. Behrens ${ }^{1} \cdot$ F. W. Schäfer ${ }^{2}$ M. van Mackelenbergh ${ }^{2} \cdot$ Christoph Röcken $^{1}(1)$
}

Received: 7 July 2021 / Accepted: 18 October 2021 / Published online: 27 October 2021

(c) The Author(s) 2021

\begin{abstract}
Background We explored the hypothesis that high-quality standards in diagnostic mammography can lead to an early diagnosis of breast cancers and identifies at risk populations outside screening programs. The histopathological features and distribution of the TNM classification were examined in relation to patient age in a large group of women with breast cancers participating in the Quality Assured Mamma Diagnostic (QuaMaDi) program of the state of Schleswig-Holstein. Patients and methods Surgical pathological reports were studied for clinicopathological characteristics, receptor status, molecular subtype and tumor stage. The analysis was conducted by dividing the study population into three age groups: women under 50 years (pre-screening), 50-69 years (peri-screening) and over 70 years (post-screening).

Results 7.111 biopsies and 2.887 resection specimens were included. Breast cancer was diagnosed in 4.241 (59.7\%) cases, one fourth of them in women $<50$ years. Elderly women ( $>70$ years) had more well-differentiated, estrogen receptor (ER)positive and HER2-negative carcinomas, whereas younger women ( $<50$ years) tended to have more poorly differentiated, ER negative, and HER2-positive carcinomas. $47 \%$ of breast carcinoma were luminal B tumors and were most common regardless of age. $70.4 \%$ of resected specimen had pT1 stage. Nodal negative were $71.2 \%$.

Conclusion In QuaMaDi breast cancer was diagnosed at an early and potentially curable stage of the disease due to highquality standards in diagnostic mammography. In addition, regardless of age, an increased number of prognostically unfavorable molecular subtypes were detected. Thus, QuaMaDi helps to identify at risk populations. QuaMaDi significantly improves diagnostic mammography and complements mammography screening programs.
\end{abstract}

Keywords Breast cancer · Quality assurance $\cdot$ Mammography $\cdot$ Immunohistochemistry $\cdot$ Molecular subtype $\cdot$ Age

\section{Introduction}

Female breast cancer is the most commonly diagnosed cancer in the world (Global Cancer Observatory 2020). Over the past decades, immense efforts have been made to improve early diagnosis and treatment. Screening programs using mammography aim to identify asymptomatic

Christoph Röcken

christoph.roecken@uksh.de

1 Department of Pathology, University Hospital SchleswigHolstein, Campus Kiel, Arnold-Heller-Str. 3, Haus U33, 24105 Kiel, Germany

2 Department of Gynecology and Obstetrics, University Hospital Schleswig-Holstein, Campus Kiel, Kiel, Germany cancer at an early disease stage, thereby reducing cancer mortality and improving life quality (Andersson et al. 1988; Andersson and Janzon 1997; Bjurstam et al. 2003; Roberts et al. 1990; Sardanelli et al. 2017). Recent European guidelines recommend the extension of mammography screening to a greater age group (European Commission Initiative on Breast Cancer 2019a, b). Nevertheless, these screening programs are not available to all women. In Germany, asymptomatic women aged between 50 and 69 years are offered a mammogram biannually (Robert Koch Institute 2016). However, women outside this age range or with breast cancer-related symptoms are excluded from the German Mammography Screening Program (GMSP) and obtain diagnostics in standard care (Institute for Cancer Epidemiology 2006). This indication-based 
mammography outside GMSP is not regulated by highquality standards implemented by the GMSP (Institute for Cancer Epidemiology 2006). Symptomatic breast cancer, often associated with a diagnostic delay, is usually associated with more aggressive tumor characteristics and therefore with a higher mortality rate than screening-detected breast cancer. In Germany, approximately $53 \%$ of incidental breast cancer cases are diagnosed outside the GMSP (Robert Koch Institute and the Association of Populationbased Cancer Registries in Germany 2019). Furthermore, half of women between 50 and 69 years do not participate in the GMSP (Cooperative Association of the German Mammography Screening Program 2019).

In Schleswig-Holstein ( $\mathrm{SH})$, the most northern federal state in Germany, the pilot-project: Quality Assured Mamma Diagnostic (QuaMaDi) was initiated in 1999 to improve the quality of indication-based breast diagnostics. QuaMaDi offers a standardized, evaluated diagnostic process including a clinical examination and an independent double reporting of mammography by two radiologists. Women who either are at risk for breast cancer, have symptoms or already have had breast cancer (after-treatment care) can participate in QuaMaDi regardless of age. If necessary, further non-invasive or invasive diagnostics (e.g., biopsy) is provided by the reference center (certified breast center) (Obi et al. 2011). Quality-standardized documentation is carried out. Details of QuaMaDi have been described elsewhere (Obi et al. 2011; Katalinic et al. 2007; Schaefer et al. 2010). Although QuaMaDi is not a screening program, it aims to identify breast cancer at an early disease stage (Institute for Cancer Epidemiology 2006). Due to excellent results within the pilot region between 2001 and 2005 QuaMaDi has been implemented in standard care in SH (Katalinic et al. 2007). Until now, no study has systematically reviewed the biopsy and resection specimens obtained within QuaMaDi. Therefore, we carried out a retrospective, single center study on all women who had participated in QuaMaDi between 2005 and 2016 at the University Hospital Schleswig-Holstein. We aimed to test the following hypothesis: (1) QuaMaDi identifies at risk populations at time; (2) it is a highly valuable additive to GMSP; and (3) biopsy specimens obtained during QuaMaDi are representative for breast lesions, which were forwarded to surgical and/or oncological treatment.

\section{Patients and methods}

\section{Ethics statement}

Our project was granted ethical clearance by the local ethics committee of the University Hospital in Kiel, Germany, in agreement with the Helsinki Declaration (D470/17).

\section{Study population}

From the electronic database of the Department of Pathology, University Hospital Schleswig-Holstein, Campus Kiel, we retrieved all patients, who were part of QuaMaDi between 01.01.2005 and 31.12.2016. Only women were included if (1) the referral indicated "QuaMaDi" and (2) a biopsy specimen was submitted to the Dept. of Pathology. Patients were excluded if they were (1) of male gender or (2) if only cytological specimens were submitted. The following patient characteristics were retrieved from the electronic database: age, gender, date of biopsy, biopsy site, number of biopsy samples, histological diagnoses, and the B-classification (since 2007). The following additional data were retrieved for non-invasive and invasive cancers: histological type of the neoplastic lesion, anatomical site, immunoreactivity score (IRS) for the estrogen (ER) and progesterone receptor (PR) (in-situ lesions and invasive cancer), and additionally Elston and Ellis grading (since 2009), HER2 status and Ki67-index (since 2012) for invasive cancers. If patients had undergone surgery at the Dept. of Gynecology and Obstetrics, University Hospital Kiel, the following data were retrieved: date of surgery, age of the patient at the time of surgery, adjuvant or neoadjuvant therapy regimen, histological tumor type, pTNM-classification, grading, and tumor regression (according to Sinn regression score, Sinn et al. 1994). All patient-related data were pseudonymized after inclusion into the study.

\section{Histology}

Tissue specimens were fixed in formalin and embedded in paraffin. Deparaffinized sections were stained with hematoxylin and eosin. The grading system of Elston and Ellis was applied since 2009 to invasive cancers (Elston and Ellis 1991). The pTNM-stage of all study patients was determined according to sixth and seventh editions of the UICC guidelines. All biopsy and resection specimens had been examined by trained and board-certified surgical pathologists. 


\section{B-classification}

According to national guidelines for the diagnosis and treatment of breast cancer, the histopathological lesions in core (NCB) and vacuum-assisted biopsies (VAB) were categorized according to the B-classification (Ellis et al. 2004). In brief: category B 1 denotes normal breast tissue or insufficient sampling, B2 benign lesions, B3 benign lesions of unknown biological potential, B4 lesions suspicious for malignancy, B5a in situ carcinomas, B5b invasive breast cancer, $\mathrm{B} 5 \mathrm{c}$ indiscernible whether non-invasive or invasive, and B5d malignant tumor of divergent histology (e.g., malignant phylloides tumor) (S1).

\section{Immunohistochemistry and in situ hybridization}

Immunohistochemical staining was done using a Bondmax automated slide staining system (Leica Biosystems, Wetzlar, Germany), the Polymer Refine Detection Kit (Leica Biosystems) and antibodies directed against ER (dilution 1:150), PR (both Leica Biosystems Newcastle, Newcastle, United Kingdom; dilution 1:100), Her2/neu (1:100) and anti-Ki-67 antibody (both Thermo Fisher Scientific, Fermont, USA; dilution 1:300). For chromogenic in situ hybridization the ZytoDot ${ }^{\circledR}$ 2C SPEC ERB2/CEN 17 Probe Kit (ZytoVision $\mathrm{GmbH}$, Bremerhaven, Germany) was used.

\section{Scoring and assessment of estrogen and progesterone receptor status}

The ER and PR status were assessed according to Remmele and Stegner (1987). In brief: Category A documented the intensity of nuclear staining as absent (0), weak (1), moderate (2) or strong (3). Category B documented the percentage of stained tumor cells as absent $(0),<10 \%(1), 10-50 \%$ (2), $51-80 \%$ (2), and $>80 \%$ positive nuclei (4). The immunoreactivity score (IRS) was calculated according to the formula: Category A (immunostaining intensity) $\times$ Category B (proportion of positive cells of the tumor). An IRS of 0 and 1 was interpreted as receptor negative. IRS $\geq 2$ were considered receptor positive. The range of 2-3 represented a weak, $4-8$ a moderately strong, and $9-12$ a strong expression of the hormone receptors. Over time the IRS was abandoned, because scores between 1 and 3 could indicate both receptor negative and positive status. Hence, the percentage of positive tumor cells in the specimen was crucial for the assessment. Since 2010, each tumor was assessed according to ASCO/CAP guidelines for ER and PR testing (Hammond et al. 2010). Hormone receptor status for ER and $P R$ was considered positive if $\geq 1 \%$ of tumor cells were immunoreactive. Breast carcinoma was hormone receptor negative if $<1 \%$ of tumor cells stained (with positive internal control). The IRS was still optionally reported in the pathological findings. Finally, the hormone receptor status was determined by using the documented IRS in the pathological report. Since an IRS of 1, 2 and 3 could indicate both a negative and positive hormone receptor status, assignment to the categories hormone receptor positive and negative was based on the pathologist's evaluation as documented in the pathology report. Ambiguous reports were re-examined during this study by a board-certified surgical pathologist. All IRS values $\geq 4$ were assigned to positive hormone receptor status.

\section{Scoring and assessment of $\mathrm{Ki}-67$}

Since 2012, the Ki-67 index was assessed according to recommendations of the International Ki-67 in Breast Cancer Working Group (Dowsett et al. 2011). After prior overview of the entire tissue specimen, the evaluation of the Ki-67 proliferation index was carried out using at least 3 highpower $(\times 40$ objective) fields. The fields were selected to reflect the staining of the whole specimen. The invasion front of the tumor was evaluated. Only nuclear staining was assessed. The Ki-67 proliferation index is defined as the percentage of positively stained cells among the total number of malignant cells scored. A minimum of 100 cells was analyzed and determined by single cell counting using a counting device. If distinct clusters of positively stained cells were seen in the preparation, they were included in the overall evaluation. Finally, the Ki-67 index was categorized into the following groups: $\leq 10 \%, 10-20 \%, 20-25 \%, 25-50 \%,>50 \%$ and unclassifiable. Ranges and no exact value of Ki-67 were assigned as unclassifiable.

\section{Scoring and assessment of HER2 status}

Scoring of each breast carcinoma was assessed on core biopsy specimens according to the ASCO/CAP recommendations for HER2 testing (Wolff et al. 2007, 2013). The immunostaining intensity and extent as well as percentage of positive tumor cells was evaluated. On slide positive controls were used in each case. During study period, the recommendations of ASCO/CAP were constantly updated.

\section{Assessment of chromogenic in situ hybridization}

If the HER2 Score was $2+$ a reflex test on the same biopsy specimen was performed using the chromogenic in situ hybridization according to the ASCO/CAP recommendations for HER2 testing (Wolff et al. 2007, 2013). The amplification was determined by examining average HER2 copy number and since 2007 the HER2/CEP 17 ratio. During study period, the recommendations of ASCO/CAP were constantly updated. The in situ hybridization status used in this study are listed. 


\section{Molecular subtypes}

The classification of the molecular subtypes was based on the St. Gallen recommendations (Goldhirsch et al. 2011) with IHC analysis of ER, PR, HER2, Ki-67 and CISH analysis of HER2. The molecular subtypes were defined as follows: luminal A (ER positive, HER2 negative, Ki-67 <20\%), luminal B (either ER positive, HER2 positive and $\mathrm{Ki}-67 \geq 20 \%$ or ER positive, HER 2 negative and Ki-67 $\geq 20 \%$ ), HER 2 positive (ER negative, HER2 positive, any Ki-67) and triple-negative (ER negative, HER2 negative, any $\mathrm{Ki}-67)$.

\section{Internal and external quality assurance schemes}

During the entire study period, the Dept. of Pathology regularly participated successfully in external quality assurance schemes. These comprised the annual participation in the Quality Assurance Initiative for Pathology (QuIP) for ER, PR, Ki-67, HER2, and HER2 CISH. The proficiency test results are published and available on the homepage of the Dept. of Pathology (https://www.patho.uni-kiel.de/krank enversorgung/qualitätssicherung). In addition, the Dept. of Pathology voluntarily participated in the HER2-Monitor of the Hannover Medical School. There, the internal positivity rate of HER2 is constantly compared with the nationwide average. Furthermore, the histological examinations were carried out by trained and board-certified surgical pathologists and any ambiguous finding was re-assessed by at least one additional board-certified surgical pathologist (four-eye principle).

\section{Statistics}

SPSS version 25.0.0.2 (IBM Corp., Armonk, NY, USA) was used for statistical analyses. To test for correlation between non-ordinal variables, we used Fisher's exact test. When testing for correlation between variables of nominal scale, we used Pearson's chi square test. We assumed a significance level of 0.05 . The $p$-values were calculated by twosided $T$-tests and a $p$-value $\leq 0.01$ was considered highly significant.

\section{Results}

\section{Characteristics of the study population}

In total, 7.111 biopsy specimens and 2.887 corresponding resection specimens obtained from 6.845 women were assigned to the QuaMaDi program. The median age of the patients was 55 years (range from 15 to 94 years for biopsy specimens; 26 to 94 years for resection specimens). 6.586 women participated in the QuaMaDi program once, 252 twice and 7 three times. In 782 (11.4\%) women, more than one biopsy (different localization within the breast or different anatomical sites) was obtained per referral. We categorized our patients into three age groups: pre- $(<50$ years $)$, peri- (50-69 years) and post-screening group ( $\geq 70$ years): $2.685(37.7 \%)$ of 7.111 histological referrals were obtained from women $<50$ years (median age 44 years; age range $15-49), 2.935$ (41.3\%) from women between 50 and 69 years (median age 60; age range 50-69), and 1.491 (21.0\%) from women $\geq 70$ years (median age 75 ; age range 70-94).

\section{Comparison of QuaMaDi patients with a single biopsy}

First, we correlated the three different age groups with various pathological patient characteristics, i.e., anatomical localization (C-code), B-category, tumor type (M-category), the presence of precursor lesions, tumor grade, receptor status and Ki-67 index and molecular subtypes in patients with a single biopsy. As summarized in Table 1 significant differences between the three age groups were found for all parameters except the Ki-67 index.

The percentage of invasive tumors (B5b) increased from $30.8 \%$ in the pre- to $73.2 \%$ in the post-screening group, while the percentage of benign lesions (B2) decreased from 50.5 to $11.7 \%$. The proportion of benign lesions with uncertain biological potential (B3) halved from 8.3 to $3.7 \%$ (Table 1). While the risk of detecting cancer increased continuously with age, a substantial number of women aged $<50$ years, i.e., $35 \%$ had an in-situ carcinoma (B5a) or an invasive carcinoma (B5b).

Invasive carcinoma NST was found most commonly in the pre-screening group (81.3\%), while invasive lobular (17.5\%) and mucinous (4.2\%) carcinomas were found most commonly in the post-screening group (Table 1). Conclusively, although invasive carcinoma NST was the most common tumor type in each age group, better differentiated carcinomas, such as mucinous carcinomas, were more likely to occur with increasing age.

Likewise, the detection rate of precursor lesions increased significantly with patient age (Table 1). $29 \%$ of the high-risk lesions and in situ carcinomas occurred in women $<50$ years. The proportion of well-differentiated carcinomas (G1) also increased with age, whereas the proportion of poorly differentiated carcinomas (G3) decreased significantly from $18.9 \%$ to $11.8 \%$ (Table 1). Regardless of age, moderately differentiated carcinoma (G2) was diagnosed in more than half of the cases. The results show that younger women $(<50$ years) were more likely to have more aggressive, poorly differentiated carcinomas. 


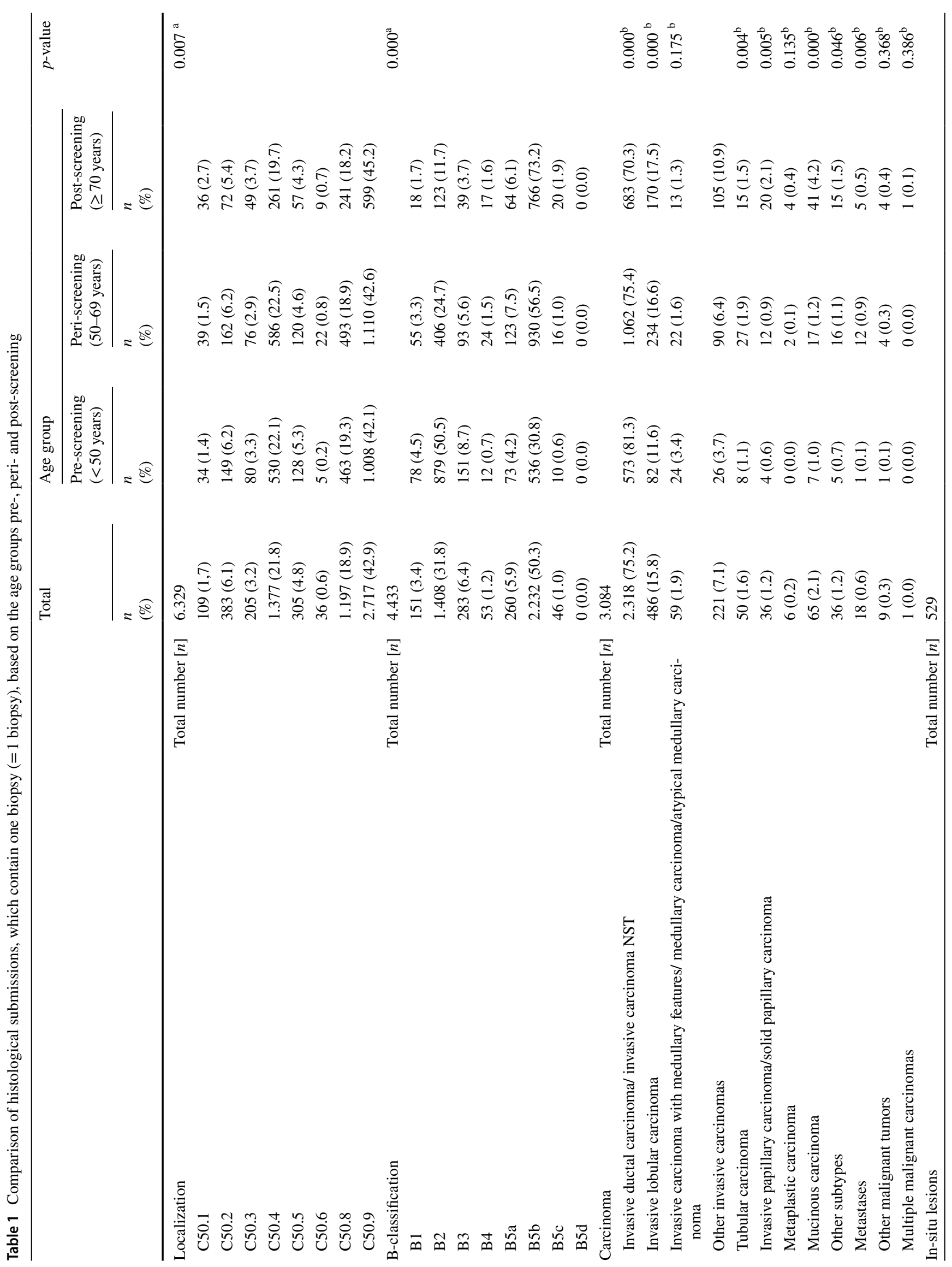




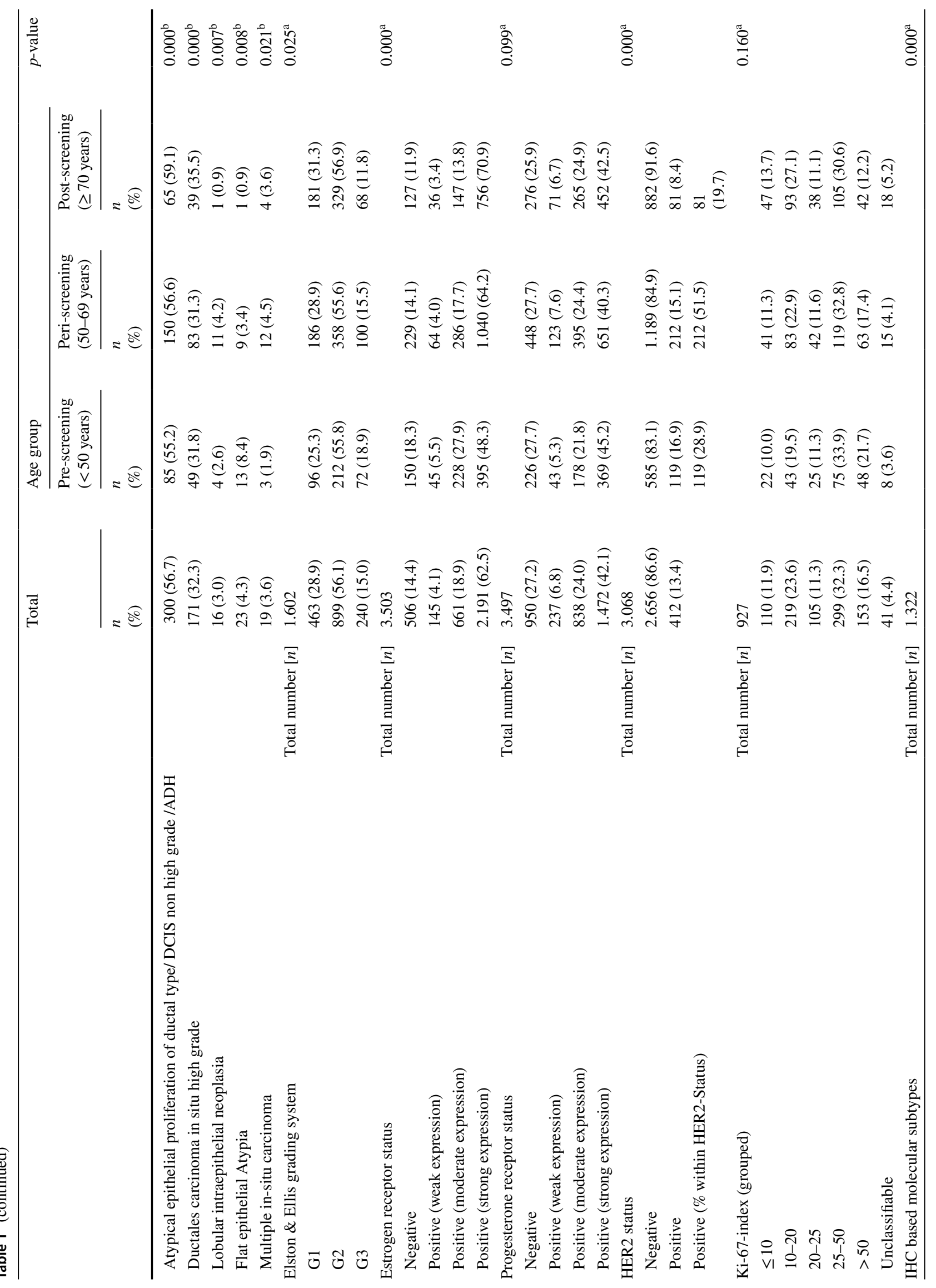




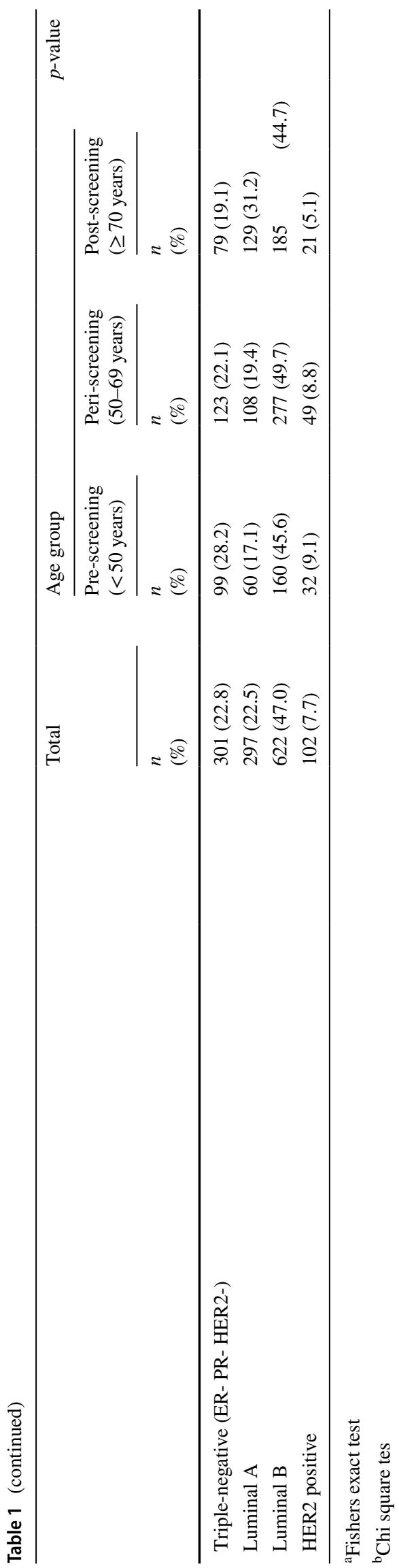

There were highly significant differences for the ER- and HER2 status: The proportion of ER-positive carcinomas increased from $81.7 \%$ in the pre- to $88.1 \%$ in the post-screening group. The percentage of HER2-positive carcinomas halved from 16.9 to $8.4 \%$. No significant differences between the three age groups were demonstrated for the PR status and Ki-67 index. Overall, the results indicate that elderly women ( $\geq 70$ years) show a higher frequency of ER positive and HER2-negative carcinomas, whereas those under 50 years tended to have ER negative and HER2-positive carcinomas, underscoring the higher frequency of more aggressive breast carcinomas in the younger age group.

The significant differences in the hormone receptor status mirrored in the four molecular subtypes, i.e., luminal A, luminal B, HER2 positive and triple-negative. The proportion of triple-negative breast carcinomas decreased significantly from $28.2 \%$ in the pre- to $19.1 \%$ in the postscreening group (Table 1). In contrast, the proportion of Luminal A carcinomas doubled from $17.1 \%$ in the pre- to $31.2 \%$ in post-screening group. Luminal B occurred most frequently in all age groups, accounting for more than $40 \%$. Overall, women $<50$ years exhibited $30.5 \%$ triple-negative and HER2-positive carcinomas.

\section{Detection frequency of malignancy in the entire cohort}

A total of 7.111 clarification diagnoses consisting of both single and multiple biopsies were categorized into the three age groups. The comparison of the three age groups showed a highly significant difference. The proportion of invasive carcinomas increased from $32.6 \%$ in the pre-screening to $74.6 \%$ in the post-screening group (S2). In particular, a linear increase of malignant breast lesions of $2.4 \%$ per year of age was observed in the age range between 43 and 77 years (Fig. 1). In addition, the ratio of benign to malignant lesions increased from an initial 1:0.64 for women $<50$ years to 1:1.99 for women between 50 and 69 years. A markedly higher ratio of benign to malignant lesions, i.e., 1:4.87, occurred for women $\geq 70$ years. Overall, $54.5 \%$ of breast carcinomas appeared beyond the screening age. In particular, $23.9 \%$ of the diagnosed carcinomas were detected in women $<50$ years.

\section{Resection specimens in patients who took part in QuaMaDi}

We identified 2.887 resection specimens of QuaMaDi, of which 2.454 cases had been assigned to primary surgical therapy (non-neo group) and 433 had received neoadjuvant therapy (neo group) (Table 2). Comparing the three age groups, we found significant differences regarding tumor type, local tumor growth (pT category), nodal spread ( $\mathrm{pN}$ 


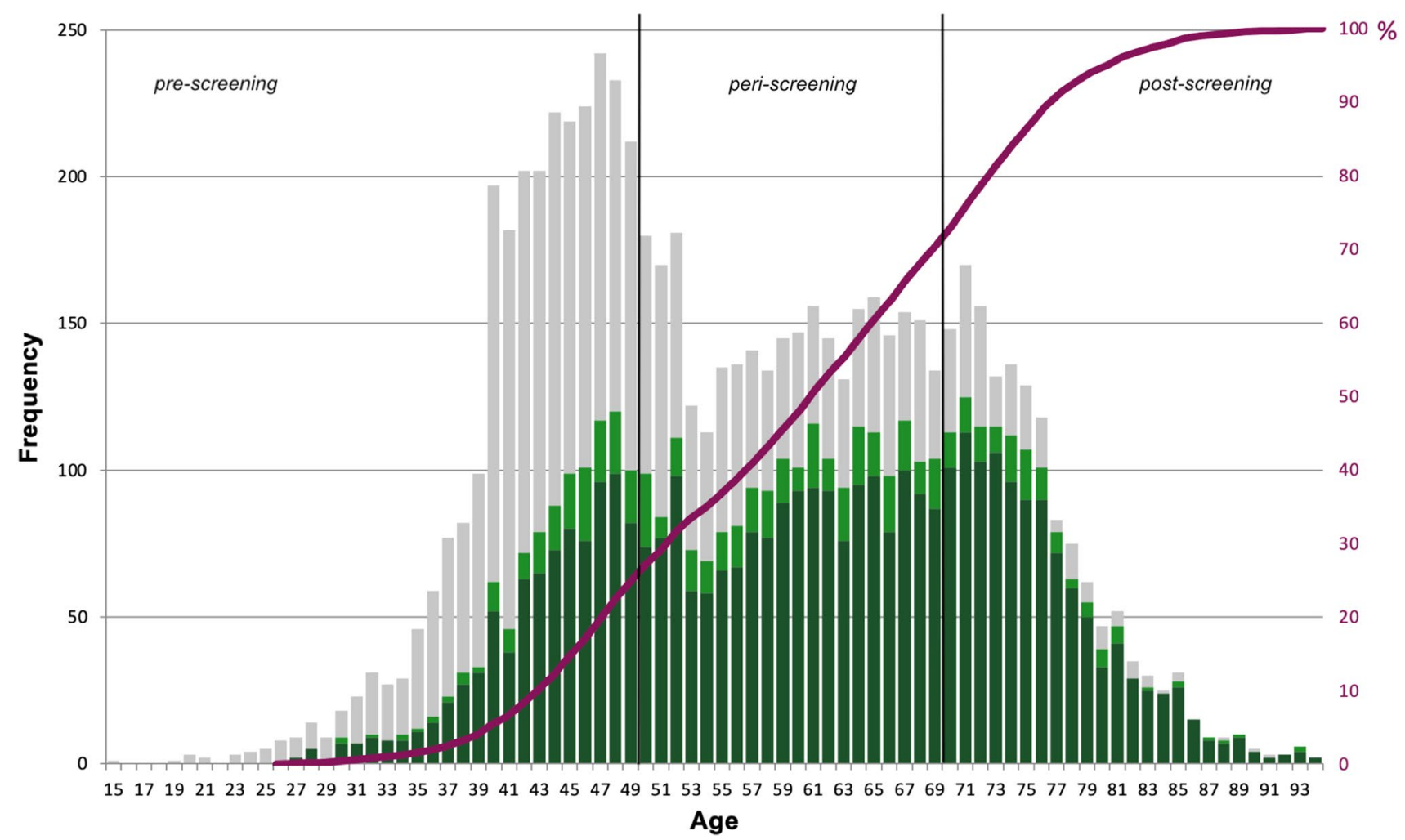

Fig. 1 Absolute age distribution of 7.111 histological submissions at the time of diagnosis in the period from $01 / 01 / 2005$ to $31 / 12 / 2016$, color-divided by the presence or absence of malignancy. The black vertical lines mark the age groups pre-, peri-, and post-screening.

category), lymph vessel invasion ( $\mathrm{pL}$ category), tumor grade and resection status (R category) in patients who were primarily resected and had not received neoadjuvant treatment (Table 2). While in women of the neo group, significant differences were only found for the pT category. In all three age groups, the tumor stage pT1c was found most commonly in the resection specimens of the non-neo group. Overall, $70.3 \%$ of the untreated carcinomas in the pre-, $75.2 \%$ in the peri- and $63.3 \%$ in the post-screening group had a tumor size $<2 \mathrm{~cm}$ (pT1). In the neoadjuvant treatment regimen, a pathologic complete remission (defined as the absence of tumor residuals in the breast and axilla) was achieved in one-fifth of the patients in both the pre- and peri-screening groups (ypT0). These results show that the majority of the carcinomas were detected at an early, more prognostically favorable stage of the disease in all three age groups. However, higher and prognostically less favorable tumor stages were found with a higher probability in women $<50$ and $\geq 70$ years.

All women who had participated in QuaMaDi presented most commonly without lymph node metastases (54.1-74.5\%; Table 2). Regarding the lymph node status,
The red curve describes the cumulative proportion of the incidence of invasive breast carcinoma and precursor lesions in relation to age at diagnosis

women in the pre-screening group were more likely to present with lymph node metastases in the non-neo group (Table 2) further substantiating that these women suffer from more aggressive tumors.

\section{Comparison between biopsy and resection specimens}

We examined the concordances of morphology in 2.561 and grading in 983 eligible cases with corresponding biopsy and resection specimens. Regarding histological diagnoses, 2.152 (84.0\%) diagnoses obtained in biopsy specimens were concordant with the diagnoses in the resection specimens. In 115 cases (4.5\%), no (invasive) carcinoma was identified in the biopsy, but invasive carcinoma was found in the resection specimen (S3). Most discordant morphologies were apparent between invasive carcinoma NST and lobular carcinoma.

Likewise, an identical tumor grade was given in 760 of $983(77.3 \%)$ corresponding biopsy and resection specimens (S4). A higher tumor grade had been documented in $40(4.0 \%)$ biopsy specimens and a lower tumor grade 


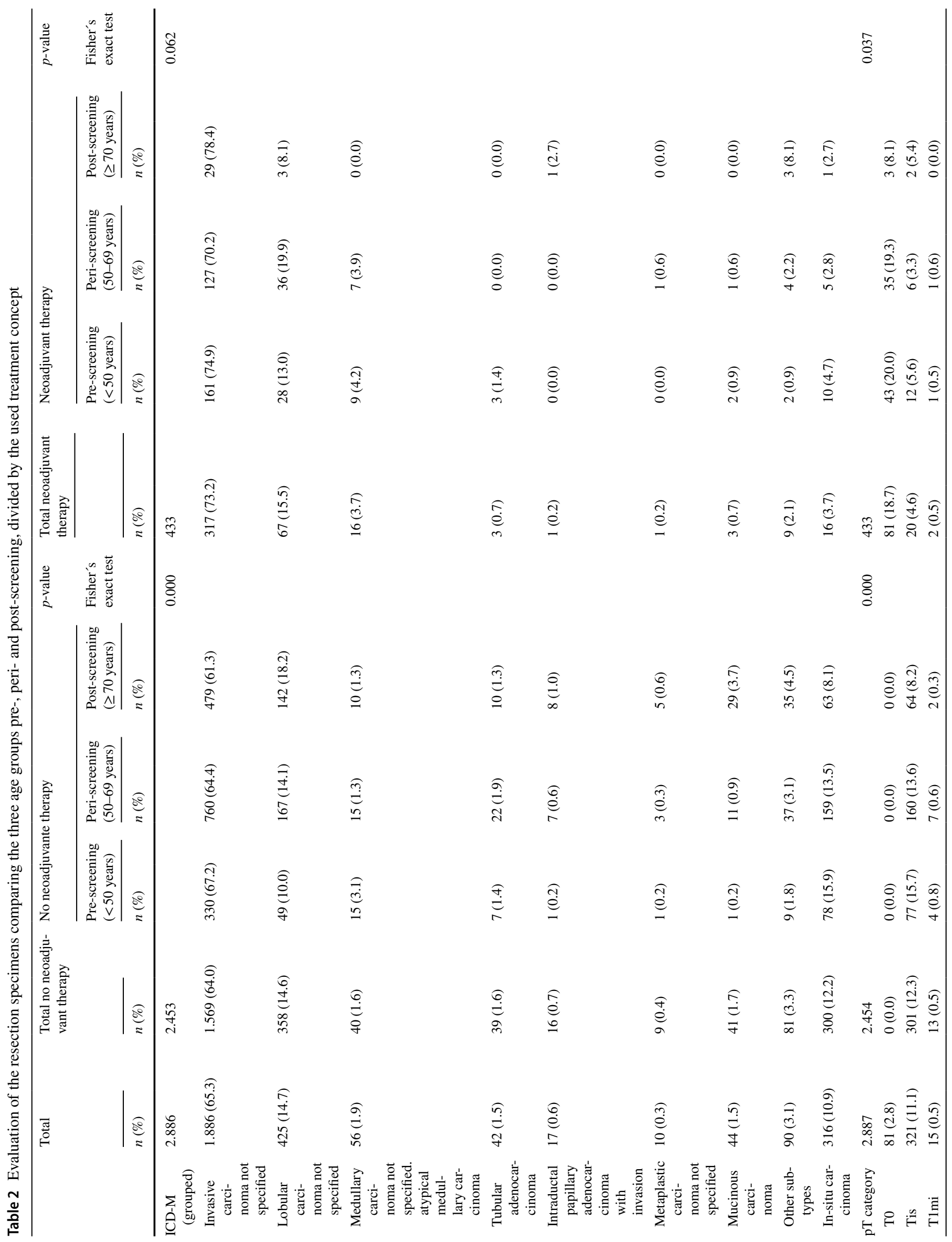




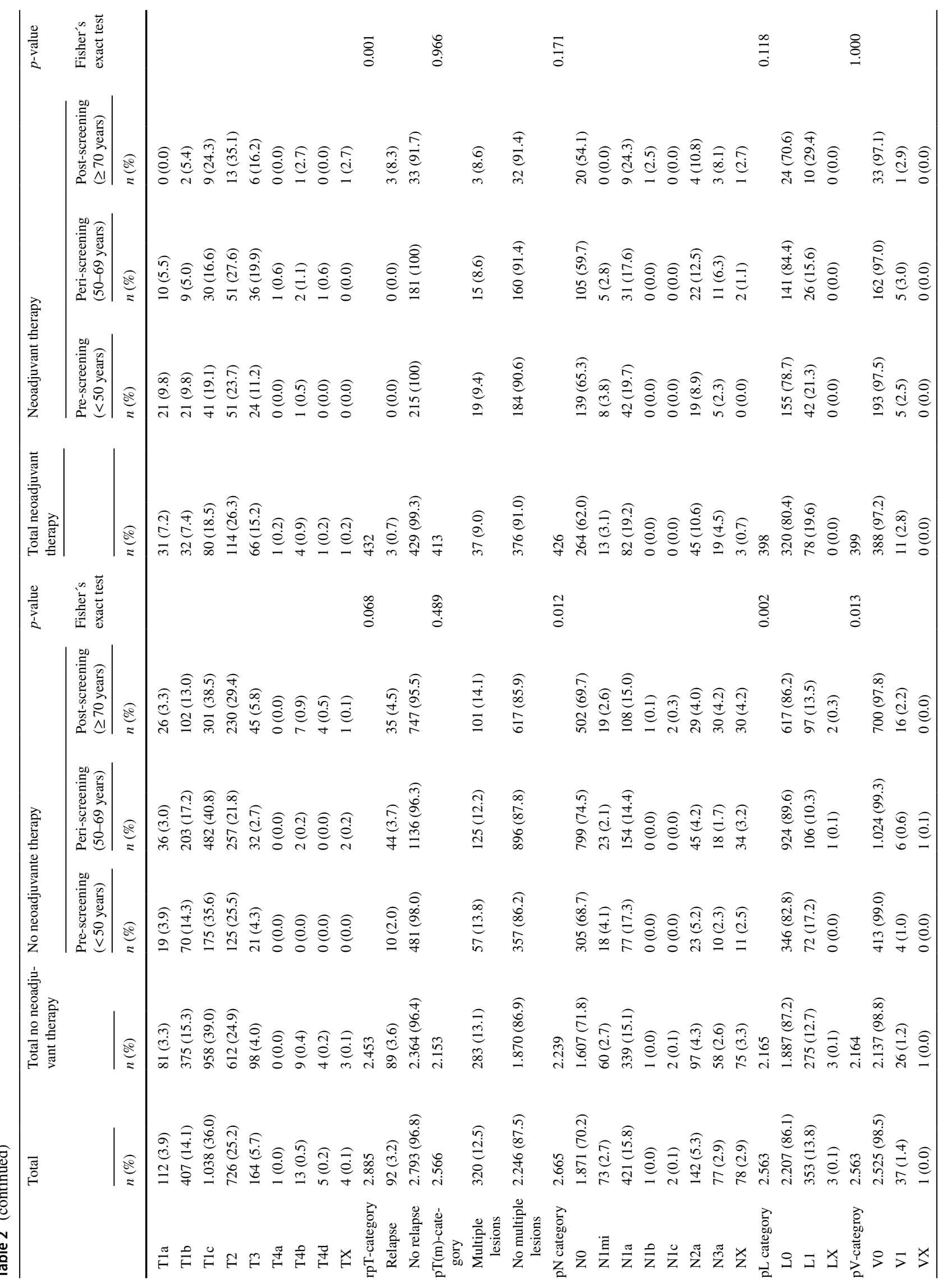




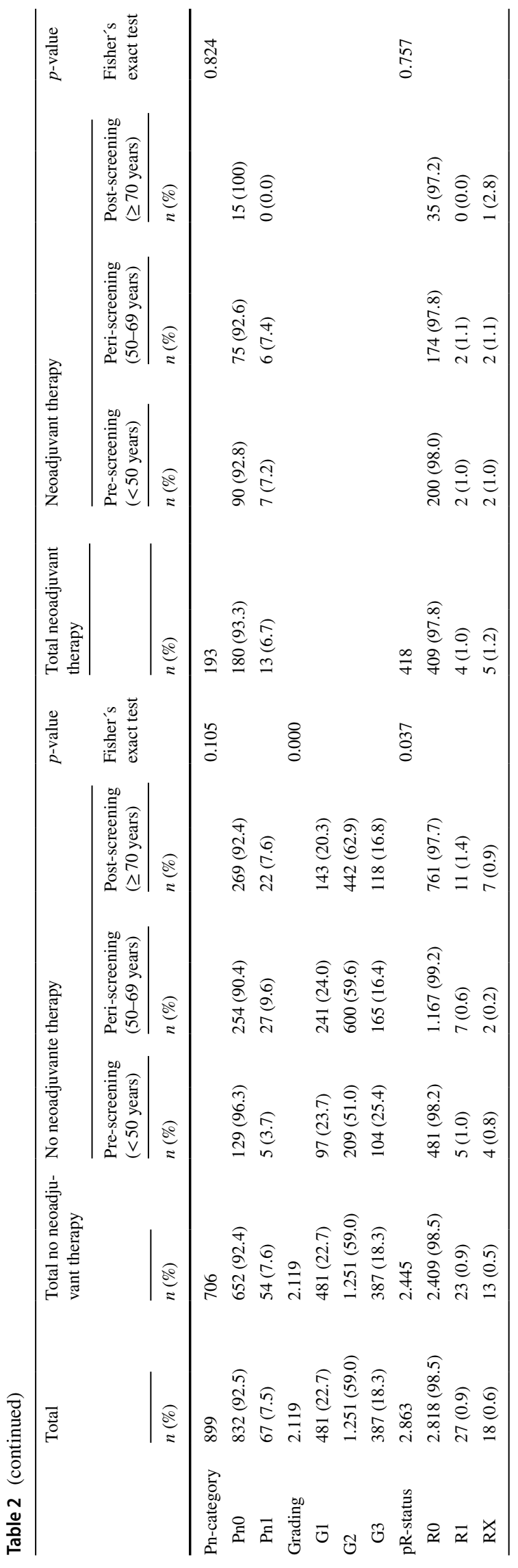

in $183(18.6 \%)$ compared with the corresponding resection specimens. Among these, two carcinomas were graded as well-differentiated carcinoma in biopsy but presented as poorly differentiated carcinoma in resection specimens. The results show that there was a tendency for undergrading in biopsies. However, overall high agreement was achieved. Tumor grading in biopsy specimens was representative in more than three quarter of the cases.

\section{Discussion}

In this retrospective study we evaluated the histopathological characteristics and TNM classification of breast carcinoma in a large cohort of women participating in the pilot-project QuaMaDi in Germany.

Our analysis highlights several significant differences when comparing the three age groups (pre-, peri-, postscreening). In particular, over one third of breast cancers diagnosed in women younger than 50 years had the molecular subtypes triple-negative and HER2 positive. In young women these molecular subtypes were associated with more aggressive tumor biology and showed marked poorer patient prognosis compared to the other subtypes (Perou et al. 2000; Sorlie et al. 2001). Additionally, the most frequent molecular subtype was luminal B in this study (between 44.7 and 49.7\%). This proportion is higher than reported in other series of breast cancer patients (range from 14 to 35\%) (Sabiani et al. 2016; Collins et al. 2012).

The higher prevalence of luminal B cancers amongst this population is concordant with the greater overexpression of Ki-67 and HER2 in tumors, both of which, in the presence of ER are characteristics of luminal B cancers (Sabiani et al. 2016). Additionally, early clinical apparent cancers are more often fast-growing carcinomas and tend more likely to be detected in diagnostic breast diagnostics as QuaMaDi. Furthermore, there are marked differences in the distribution of molecular subtypes in screening programs compared to symptomatic carcinoma (Falck et al. 2016). In concordance with our data, symptomatic patients were diagnosed with more non-luminal-A tumors (Falck et al. 2016). Furthermore, tumors of elderly patients showed more ER positivity and less overexpression of HER2 and Ki-67 (Sabiani et al. 2016; Falck et al. 2016). In line with this, the incidence of triple-negative carcinomas decreased with age, as well as HER2 overexpression. The high proportion of HER2 positive and triple-negative carcinomas in women younger than 50 years indicates that QuaMaDi can identify an at risk population.

Among others the proportion of diagnosed in-situ carcinoma is used as an evaluation parameter in screening programs. According to EU guideline the proportion 
of in-situ carcinoma in screening programs should be above 15\% (European Reference Organisation for Quality Assured Breast Screening and Diagnostic Services 2006). Although QuaMaDi is not a screening program, we achieved this goal and found in situ carcinomas in $16.7 \%$ of women $<50$ years and in $15.4 \%$ of women between 50 and 69 years. Nevertheless, screening programs reach higher proportions by approximately $20 \%$ (Cooperative Association of the German Mammography Screening Program 2018; The National Health Service Breast Screening Programme 2021). The different inclusion criteria of QuaMaDi, where symptomatic women are included, may partly explain this difference. It is known that only about $20 \%$ of in-situ carcinoma become symptomatic, whereas $80 \%$ are asymptomatic and are more often detected in screening programs (Virnig et al. 2010). Furthermore, symptomatic in situ carcinomas have an increased risk of recurrence compared to lesions detected by screening (Shamliyan et al. 2010). Especially younger women $(<50$ years) represent a high-risk population. In our cohort $29 \%$ of high-risk lesions and in situ carcinomas were diagnosed in younger women $(<50$ years), leading to early diagnosis and identification of high-risk patients.

The majority of breast carcinoma in QuaMaDi was diagnosed at an early disease stage, i.e., $60.0-71.4 \%$ with pT1 in our cohort compared with $52 \%$ in the national epidemiological data set (Robert Koch Institute 2016). Previous studies already described the positive impact of QuaMaDi (Robert Koch Institute 2016; Obi et al. 2011). Although, length time bias can influence the distribution of tumor stage (Falck et al. 2016; Lawrence et al. 2009). Tumors diagnosed by screening programs are more often slow growing, less aggressive tumors and show more favorable tumor biology (Falck et al. 2016). The less favorable stage distribution in elderly patients is associated with fewer consultation of gynecologist for screenings or by less breast cancer awareness (Obi et al. 2011). This could lead to a delayed diagnosis of advanced disease. In addition, lead time bias must be considered. Due to the study design, length and lead time bias cannot be excluded. Nevertheless, breast carcinomas were diagnosed with less favorable and more aggressive tumor biology in our cohort. The more aggressive tumor biology, particularly in women of young disease age, could explain the lower proportion of pT1 tumors compared to women in the peri-screening age group. The overall higher proportion of pT1 tumors compared to national data indicates that QuaMaDi contributes to improved medical care for women who are unable or decline to participate in the GMSP (Obi et al. 2011; Katalinic et al. 2007).

Nodal involvement also revealed age-specific differences. We noted a decrease in nodal positive breast cancers by age 70 and an additional increase in women older than 70 years. The more frequent detection of breast carcinoma in advanced tumor stages in older women as well as the agerelated decrease of immunological defense function, which favors invasion and nodal involvement are possible explanations (Daidone et al. 2003; Larbi et al. 2008; Wildiers et al. 2009). The high proportion of node negative breast carcinomas may indicate that despite the more aggressive tumor biology, breast carcinomas were diagnosed at a nonmetastatic stage of disease.

Evaluating the agreement between biopsy and surgical specimen: $77.3 \%$ of cases agreed in grading and $84 \%$ were concordant in morphology. These proportions are in line to the one reported in other studies (O'Leary et al. 2004; Rakha and Ellis 2007; The Royal College of Pathologists 2016) and confirm that biopsies obtained within QuaMaDi are representative for resected specimens. The surgical materials tended to be higher grade than biopsies. Influencing factors of disagreement might be insufficient sample volume or the invasion front was not covered, so that a different value could emerge in the resected specimens (O'Leary et al. 2004). Discrepancy in morphology could be influenced by several factors: sample volume, differentiation of the tumor and regressive changes after neoadjuvant therapy. However, the influencing factors also affect screening biopsies.

Limitations of our study include its retrospective character. Furthermore, the data was based on the pathology reports of only one of four reference centers in QuaMaDi. Nevertheless, a high number of biopsies and resected specimens were included and emphasize the representativeness of our results. Missing TNM classification of all diagnosed breast carcinoma is another limitation and is due to data protection guidelines in Germany. This may have influenced the distribution of tumor and nodal stages in this study. Also, modified immunohistochemical staining protocols as well as updated international recommendations of receptor positivity or negativity are influencing factors. Although, we achieved the expected positivity rates for ER $(\sim 80 \%)$, PR ( 60-70\%), and HER2 ( 15\%) (Lakhani et al. 2012). Internal and external quality controls during study period ensure the representativeness and validity of the results. Nevertheless, interobserver variability cannot be excluded. But, the different evaluation algorithms affected all three age cohorts equally in the respective period.

\section{Conclusion}

In conclusion, high-quality standards in standard care of breast diagnostics leads to an early diagnosis of breast cancer. Even if QuaMaDi cannot be compared to mammography screening, their effects on tumor stage distribution and nodal involvement are similar. Among our large cohort of women, 
clinicopathological features and molecular phenotypes were different across age groups. In particular, $1 / 4$ of breast cancers were diagnosed in women under 50 years and regardless of age, the tumors showed more aggressive, prognostically unfavorable tumor characteristics. Therefore, risk population could be identified. Considering additionally that $60 \%$ (Schaefer et al. 2010) of all breast cancers are diagnosed within standard care, the implementation of a high-quality breast diagnostics, as provided in QuaMaDi, can improve and complement breast cancer care.

Supplementary Information The online version contains supplementary material available at https://doi.org/10.1007/s00432-021-03841-x.

\section{Acknowledgements None.}

Funding Open Access funding enabled and organized by Projekt DEAL. None.

\section{Declarations}

Conflict of interest M. van Mackelenbergh declares to have the following conflicts of interest: Personal Fees, Honoraria or Travel grants from Amgen, AstraZeneca, Daichii Sankyo, GenomicHealth, Lilly, Mylan, Novartis, Pfizer, PierreFabre, Roche and Seagen. All remaining authors have declared no conflicts of interest.

Open Access This article is licensed under a Creative Commons Attribution 4.0 International License, which permits use, sharing, adaptation, distribution and reproduction in any medium or format, as long as you give appropriate credit to the original author(s) and the source, provide a link to the Creative Commons licence, and indicate if changes were made. The images or other third party material in this article are included in the article's Creative Commons licence, unless indicated otherwise in a credit line to the material. If material is not included in the article's Creative Commons licence and your intended use is not permitted by statutory regulation or exceeds the permitted use, you will need to obtain permission directly from the copyright holder. To view a copy of this licence, visit http://creativecommons.org/licenses/by/4.0/.

\section{References}

Andersson I, Janzon L (1997) Reduced breast cancer mortality in women under age 50: updated results from the Malmö Mammographic Screening Program. J Natl Cancer Inst Monogr. https:// doi.org/10.1093/jncimono/1997.22.63

Andersson I, Aspegren K, Janzon L, Landberg T, Lindholm K, Linell F et al (1988) Mammographic screening and mortality from breast cancer: the Malmö mammographic screening trial. BMJ 297:943948. https://doi.org/10.1136/bmj.297.6654.943

Bjurstam N, Björneld L, Warwick J, Sala E, Duffy SW, Nyström L et al (2003) The Gothenburg Breast Screening Trial. Cancer 97:23872396. https://doi.org/10.1002/cncr.11361

Collins LC, Marotti JD, Gelber S, Cole K, Ruddy K, Kereakoglow $S$ et al (2012) Pathologic features and molecular phenotype by patient age in a large cohort of young women with breast cancer. Breast Cancer Res Treat 131:1061-1066. https://doi.org/10.1007/ s10549-011-1872-9
Cooperative Association of the German Mammography Screening Program (2018) Annual report Evaluation 2016. The German Mammography Screening Program, https://fachservice.mammo-progr amm.de/download/downloads/KOOPMAMMO_Jahresbericht_ Eval_2016_20191114_web.pdf. Accessed 10 Apr 2019

Cooperative Association of the German Mammography Screening Program (2019) Annual report Evaluation 2017. The German Mammography Screening Program, https://fachservice.mammo-progr amm.de/download/downloads/KOOPMAMMO_Jahresbericht_ Eval_2017_20191114_web.pdf. Accessed 21 June 2020

Daidone MG, Coradini D, Martelli G, Veneroni S (2003) Primary breast cancer in elderly women: biological profile and relation with clinical outcome. Crit Rev Oncol Hematol 45:313-325. https://doi.org/10.1016/S1040-8428(02)00144-0

Dowsett M, Nielsen TO, A'Hern R, Bartlett J, Coombes RC, Cuzick J et al (2011) Assessment of Ki67 in breast cancer: recommendations from the International Ki67 in Breast Cancer working group. J Natl Cancer Inst 103:1656-1664. https://doi.org/10.1093/jnci/ djr393

Ellis IO, Humphreys S, Michell M, Pinder SE, Wells CA, Zakhour HD et al (2004) Best Practice No 179. Guidelines for breast needle core biopsy handling and reporting in breast screening assessment. J Clin Pathol 57:897-902. https://doi.org/10.1136/jcp.2003. 010983

Elston CW, Ellis IO (1991) Pathological prognostic factors in breast cancer. I. The value of histological grade in breast cancer: experience from a large study with long-term follow-up. Histopathology 19:403-410. https://doi.org/10.1111/j.1365-2559.1991.tb00229.x

European Reference Organisation for Quality Assured Breast Screening and Diagnostic Services (2006) European guidelines for quality assurance in breast cancer screening and diagnosis, https://www. euref.org/downloads?download=24: european-guidelines-forquality-assurance-in-breast-cancer-screening-and-diagnosis-pdf. Accessed 27 Sepr 2019

European Commission Initiative on Breast Cancer (ECIBC) (2019a) European guidelines on breast cancer screening and diagnosis in women aged 45 to 49 , https://healthcare-quality.jrc.ec.europa.eu/ sites/default/files/Guidelines/EtDs/ECIBC_GLs_EtD_screening_ 45-49.pdf. Accessed 30 June 2021

European Commission Initiative on Breast Cancer (ECIBC) (2019b) European guidelines on breast cancer screening and diagnosis in women aged 70 to 74 , https://healthcare-quality.jrc.ec.europa.eu/ sites/default/files/Guidelines/EtDs/ECIBC_GLs_EtD_screening_ 70-74.pdf. Accessed 30 June 2021

Falck AK, Röme A, Fernö M, Olsson H, Chebil G, Bendahl PO et al (2016) St Gallen molecular subtypes in screening-detected and symptomatic breast cancer in a prospective cohort with longterm follow-up. Br J Surg 103:513-523. https://doi.org/10.1002/ bjs. 10070

Global Cancer Observatory (2020) Cancer Tomorrow. International Agency for Research on Cancer, https://gco.iarc.fr/today/data/ factsheets/cancers/39-All-cancers-fact-sheet.pdf. Accessed 15 Mar 2021

Goldhirsch A, Wood WC, Coates AS, Gelber RD, Thürlimann B, Senn HJ et al (2011) Strategies for subtypes-dealing with the diversity of breast cancer: highlights of the St. Gallen International Expert Consensus on the Primary Therapy of Early Breast Cancer 2011. Ann Oncol 22:1736-1747. https://doi.org/10.1093/annonc/ mdr304

Hammond ME, Hayes DF, Dowsett M, Allred DC, Hagerty KL, Badve S et al (2010) American Society of Clinical Oncology/College Of American Pathologists guideline recommendations for immunohistochemical testing of estrogen and progesterone receptors in breast cancer. J Clin Oncol 28:2784-2795. https://doi.org/10. 1200/JCO.2009.25.6529 
Institute for Cancer Epidemiology (2006) Evaluation of quality assurance in breast cancer diagnosis (QuaMaDi): Final report 2001 to 2005 , http://www.quamadi.de/ergebnisse/, http://www.kvsh. de/admin/ImageServer.php?download=true \&ID=117@KVSH Accessed 7 Oct 2018

Katalinic A, Bartel C, Raspe H, Schreer I (2007) Beyond mammography screening: quality assurance in breast cancer diagnosis (The QuaMaDi Project). Br J Cancer 96:157-161. https://doi.org/10. 1038/sj.bjc.6603506

Lakhani SR, Ellis IO, Schnitt SJ (2012) WHO classification of tumours of the breast, 4th edn. IARC Press, Lyon

Larbi A, Franceschi C, Mazzatti D, Solana R, Wikby A, Pawelec G (2008) Aging of the immune system as a prognostic factor for human longevity. Physiology (bethesda) 23:64-74. https://doi.org/ 10.1152/physiol.00040.2007

Lawrence G, Wallis M, Allgood P, Nagtegaal ID, Warwick J, Cafferty FH et al (2009) Population estimates of survival in women with screen-detected and symptomatic breast cancer taking account of lead time and length bias. Breast Cancer Res Treat 116:179-185. https://doi.org/10.1007/s10549-008-0100-8

Obi N, Waldmann A, Schäfer F, Schreer I, Katalinic A (2011) Impact of the Quality assured Mamma Diagnostic (QuaMaDi) programme on survival of breast cancer patients. Cancer Epidemiol 35:286292. https://doi.org/10.1016/j.canep.2010.09.001

O'Leary R, Hawkins K, Beazley JC, Lansdown MR, Hanby AM (2004) Agreement between preoperative core needle biopsy and postoperative invasive breast cancer histopathology is not dependent on the amount of clinical material obtained. J Clin Pathol 57:193-195. https://doi.org/10.1136/jcp.2003.12914

Perou CM, Sorlie T, Eisen MB, van de Rijn M, Jeffrey SS, Rees CA et al (2000) Molecular portraits of human breast tumours. Nature 406:747-752. https://doi.org/10.1038/35021093

Rakha EA, Ellis IO (2007) An overview of assessment of prognostic and predictive factors in breast cancer needle core biopsy specimens. J Clin Pathol 60:1300-1306. https://doi.org/10.1136/jcp. 2006.045377

Remmele W, Stegner HE (1987) Recommendation for uniform definition of an immunoreactive score (IRS) for immunohistochemical estrogen receptor detection (ER-ICA) in breast cancer tissue. Pathologe 8:138-140 (PMID: 3303008)

Robert Koch Institute (2016) Center for Cancer Registry Data: Report on cancer events in Germany 2016, https://edoc.rki.de/bitstream/ handle/176904/3264/28oaKVmif0wDk.pdf?sequence=1\&isAll owed=y;. Accessed 1 June 2018

Robert Koch Institute and the Association of Population-based Cancer Registries in Germany (2019) Cancer in Germany 2015/2016, https://www.krebsdaten.de/Krebs/DE/Content/Publikationen/ Krebs_in_Deutschland/kid_2019/krebs_in_deutschland_2019. pdf?_blob=publicationFile. Accessed 24 May 2020

Roberts MM, Alexander FE, Anderson TJ, Chetty U, Donnan PT, Forrest $P$ et al (1990) Edinburgh trial of screening for breast cancer: mortality at seven years. Lancet 335:241-246. https://doi.org/10. 1016/0140-6736(90)90066-E

Sabiani L, Houvenaeghel G, Heinemann M, Reyal F, Classe JM, Cohen $\mathrm{M}$ et al (2016) Breast cancer in young women: Pathologic features and molecular phenotype. Breast 29:109-116. https://doi.org/10. 1016/j.breast.2016.07.007

Sardanelli F, Aase HS, Álvarez M, Azavedo E, Baarslag HJ, Balleyguier C et al (2017) Position paper on screening for breast cancer by the European Society of Breast Imaging (EUSOBI) and 30 national breast radiology bodies from Austria, Belgium, Bosnia and Herzegovina, Bulgaria, Croatia, Czech Republic, Denmark, Estonia, Finland, France, Germany, Greece, Hungary, Iceland, Ireland, Italy, Israel, Lithuania, Moldova, The Netherlands, Norway, Poland, Portugal, Romania, Serbia, Slovakia, Spain, Sweden, Switzerland and Turkey. Eur Radiol 27:2737-2743. https://doi. org/10.1007/s00330-016-4612-z

Schaefer FK, Waldmann A, Katalinic A, Wefelnberg C, Heller M, Jonat $\mathrm{W}$ et al (2010) Influence of additional breast ultrasound on cancer detection in a cohort study for quality assurance in breast diagnosis-analysis of 102,577 diagnostic procedures. Eur Radiol 20:1085-1092. https://doi.org/10.1007/s00330-009-1641-x

Shamliyan T, Wang SY, Virnig BA, Tuttle TM, Kane RL (2010) Association between patient and tumor characteristics with clinical outcomes in women with ductal carcinoma in situ. J Natl Cancer Inst Monogr. https://doi.org/10.1093/jncimonographs/lgq034

Sinn HP, Schmid H, Junkermann H, Huober J, Leppien G (1994) Histologic regression of breast cancer after primary (neoadjuvant) chemotherapy. Geburtshilfe Frauenheilkd 54:552-558. https://doi. org/10.1055/s-2007-1022338

Sorlie T, Perou CM, Tibshirani R, Aas T, Geisler S, Johnsen H et al (2001) Gene expression patterns of breast carcinomas distinguish tumor subclasses with clinical implications. Proc Natl Acad Sci USA 98:10869-10874. https://doi.org/10.1073/pnas.191367098

The National Health Service Breast Screening Programme (2021) NHS Breast Screening Programme in England in 2019-20, https://files. digital.nhs.uk/F9/98C8E3/breast-screening-programme-eng2019-20-report.pdf. Accessed 10 Apr 2021

The Royal College of Pathologists (2016) Guidelines for non-operative diagnostic procedures and reporting in breast cancer screening. https://www.rcpath.org/uploads/assets/4b16f19c-f7bd-456c-b212f 557f8040f66/G150-Non-op-reporting-breast-cancer-screeningFeb17.pdf. Accessed 13 Apr 2019

Virnig BA, Tuttle TM, Shamliyan T, Kane RL (2010) Ductal carcinoma in situ of the breast: a systematic review of incidence, treatment, and outcomes. J Natl Cancer Inst 102:170-178. https://doi. org/10.1093/jnci/djp482

Wildiers H, Van Calster B, van de Poll-Franse LV, Hendrickx W, Roislien J, Smeets A et al (2009) Relationship between age and axillary lymph node involvement in women with breast cancer. J Clin Oncol 27:2931-2937. https://doi.org/10.1200/JCO.2008.16.7619

Wolff AC, Hammond ME, Schwartz JN, Hagerty KL, Allred DC, Cote RJ et al (2007) American Society of Clinical Oncology/College of American Pathologists guideline recommendations for human epidermal growth factor receptor 2 testing in breast cancer. J Clin Oncol 25:118-145. https://doi.org/10.1200/JCO.2006.09.2775

Wolff AC, Hammond ME, Hicks DG, Dowsett M, McShane LM, Allison KH et al (2013) Recommendations for human epidermal growth factor receptor 2 testing in breast cancer: American Society of Clinical Oncology/College of American Pathologists clinical practice guideline update. J Clin Oncol 31:3997-4013. https://doi.org/10.1200/JCO.2013.50.9984

Publisher's Note Springer Nature remains neutral with regard to jurisdictional claims in published maps and institutional affiliations. 